\title{
An emergent universe supported by a Nonlinear Sigma Model
}

\author{
A. Beesham* \\ Department of Mathematical Sciences, Zululand University \\ Private Bag X1001 \\ Kwa-Dlangezwa 3886, South Africa \\ S. V. Chervon ${ }^{\dagger}$ and S. D. Maharaj ${ }^{\ddagger}$ \\ Astrophysics and Cosmology Research Unit \\ School of Mathematical Sciences, University of KwaZulu-Natal \\ Private Bag X54 001 \\ Durban 4000, South Africa
}

November 1, 2018

\begin{abstract}
We suggest the use of a nonlinear sigma model as the source which supports an emergent universe. The two-component nonlinear sigma model is considered as the simplest model containing inflaton and auxiliary chiral fields.
\end{abstract}

\section{Introduction}

The emergent universe (EmU) scenario was originally proposed by Ellis and Maartens in 2002 [1] with the aim of avoiding the Big Bang singularity, and to open up the question about the existence of quantum gravity. The EmU scenario has been accepted by many cosmologists 2, 3, 4, as a viable model, and has been extended in many aspects. Some of the recent treatments include the scalar-tensor theory of gravity [5] and brane world gravity [6].

In the original EmU model, Ellis and Maartens suggested the existence of ordinary matter with energy density $\rho$ and pressure $p=w \rho$. It is difficult to imagine such matter when $t \rightarrow-\infty$, because of the very small volume of the universe with the radius $a_{i}$, which is not very much larger than the Plank scale. Therefore in the present article we suggest that this "ordinary matter" be replaced with the chiral fields in the framework of the non-linear sigma model (NSM). Chiral NSM were introduced as a theory of strong interactions at the end of the fifties by Schwinger [7] and Skyrme [8]. Gell-Mann and Levy

\footnotetext{
*E-mail: abeesham@pan.uzulu.ac.za

${ }^{\dagger}$ E-mail: chervons@ukzn.ac.za (On leave from Department of Theoretical Physics, Ulyanovsk State University, Ulyanovsk, 432700 Russia)

${ }^{\ddagger}$ E-mail: maharaj@ukzn.ac.za
} 
in their work 9 pointed out how to realize the chiral symmetry and partial conservation of the axial vector current. This article is often referred as the the work where the terms "linear and nonlinear sigma model" have been introduced. The investigation of the mathematical aspects of the NSM was closely connected with the two dimensional version of the model because of their analogy in many respects to non-Abelian gauge theories. The main results of this investigations, such as soliton, instanton and meron solutions, as well as the application of the inverse scattering method application are summed up in the review [10.

The consistent construction of the four dimension NSM can be realized only by including a coupling to the gravitational field, as was found when instanton solutions were investigated [11. Consideration of NSM as the source of the gravitational field (with Lorenz signature) was proposed by G. Ivanov 12 (see also [13). The applications of the chiral NSM in general relativity and cosmology have been examined also in [14, 15. In particular, it was shown that chiral NSM with a potential of self-interaction (called in the framework of cosmology - chiral cosmological model) contains the self-interacting scalar field (SSF) theories as well as multicomponent ones. Therefore the chiral NSM can be considered as an effective model and can describe not only SSF theory [16, but some classes of the gravitational field as well [17.

Thus we can see the advantages and perspectives for the application of the chiral NSMs to the EmU because of its wide physical content and the fact that various geometrical methods can be applied in its investigation.

Now we can apply the NSM as the source which support the EmU from the very beginning (in description of negative times) to late times. We shall start from the two component NSM as the simplest possibility to find the solutions describing the features of the model. The first scalar field we will consider is the inflaton while an additional chiral field in this case may have various physical interpretations. It may be the field inspired by superstring cosmology [18, such as the dilaton or moduli fields, or it may be the scalar field responsible for dark matter or dark energy [19]. After studying the model with two fields, it will then be possible to introduce more then one auxiliary chiral field with the aim of considering interactions between dark matter, dark energy and the inflaton as the source of the EmU.

There are a few approaches that we could follow in investigating issues relevant in cosmology for NSM and SSF theory. The first approach is the direct calculation with a given potential [15]. In this approach we believe that high energy physics (HEP) may provide us with the form of the potential $V$ of selfinteraction for the scalar field as the function on $\phi: V=V(\phi)$. Then this dependence closes the set of Einstein and scalar field equations, and we have to solve two equations with two unknown functions: scalar field and the scalar factor as the functions of cosmic time. The second approach which is also connected with HEP is the fine turning method with a given scalar field evolution $\phi=\phi(t)$ [20]. In this approach the dependence of scalar field on cosmic time is considered as given. This fact also closes the set of equations and we can find, from Einstein and scalar field equations, the scalar factor and the potential of self-interaction as the function of cosmic time. The third approach (the fine tuning of the potential method) is based on astrophysical observations of the evolution of the scalar factor. We can put as a physical reality the evolution of scalar factor $a=a(t)$ into the set of Einstein and scalar field equations. Then we can find the potential $V(t)$ and scalar field evolution $\phi=\phi(t)$. This method 
has been proposed in 21 and developed in 22, 23. The investigation in EmU is connected with the third approach, when the evolution of the scalar factor is given a priori. Considering the fine tuning of the potential method we can investigate matter which is responsible for the evolution of the Universe that we observe. It is very important to understand what types of matter manage the expansion of the Universe and lie beyond this expansion. It is almost evident that it may be various types of matter (equations of state) that support observable expansion. The fact that NSM can provide support for the same gravitational field, as SSF theory, has been proved earlier [13. Moreover it was found that the effective scalar field represents NSM in the Einstein equations [15. In this case we have to solve the dynamic equations for the chiral fields. This result means that a single effective scalar field may contain a great number of chiral fields, which can represent dark matter, dark energy, phantom and quintessence fields, etc. These fields, hidden in one effective scalar field, can exhibit themselves in dynamical equations. Therefore it is of great importance to study the chiral field equations because their solutions will give new light for the better understanding of the physical nature of hidden fields. There are other special methods of exact cosmological solution construction in SSF theory such as generation of new solutions with fine turned potential $V=V(t)[23$, comparative analysis of slow-roll and exact solutions in inflationary models 24$]$ which lie outside our consideration here.

The article is organized as follow. First, we introduce the main equations of a self-gravitating NSM. In the next section we consider the chiral cosmological model for the two component NSM. The dynamical evolution of the chiral fields around the minimum of the scale factor is considered for the first time in the EmU. We present an example of the solution and investigate its asymptotic behaviour when $t \rightarrow-\infty$. In Section 5 , we investigate the evolution of the chiral fields at late times. The two exact solutions that we obtain involve new types of potential which support the EmU at late times. We discuss our results in the last section.

\section{A self-gravitating NSM}

The action for a self-gravitating NSM endowed with a self-interacting potential $W(\varphi)$ is given by [14, 25 .

$$
\mathcal{S}=\int \sqrt{-g} d^{4} x\left\{\frac{R}{2 \kappa}+\frac{1}{2} h_{A B}(\varphi) \varphi_{, \mu}^{A} \varphi_{, \nu}^{B} g^{\mu \nu}-W(\varphi)\right\}
$$

Here $g_{\mu \nu}(x)$ is the space-time metric, and $h_{A B}(\varphi)$ is the metric of the target space, $\varphi=\left(\varphi^{1}, \ldots, \varphi^{n}\right), \partial_{\mu} \varphi^{A}=\varphi_{, \mu}^{A}$.

For the chiral cosmological model, the energy-momentum tensor corresponding to the action (11) reads

$$
T_{\mu \nu}=\varphi_{A, \mu} \varphi_{, \nu}^{A}-g_{\mu \nu}\left\{\frac{1}{2} \varphi_{, \alpha}^{A} \varphi_{, \beta}^{B} g^{\alpha \beta} h_{A B}-W\left(\varphi^{C}\right)\right\} .
$$

Einstein's equations can be transformed to

$$
R_{\mu \nu}=\kappa\left\{h_{A B} \varphi_{, \mu}^{A} \varphi_{, \nu}^{B}-g_{\mu \nu} W\left(\varphi^{C}\right)\right\}
$$


By varying the action (11) with respect to the chiral fields $\varphi^{C}$, we can obtain the equations of motion of the chiral fields as

$$
\frac{1}{\sqrt{-g}} \partial_{\mu}\left(\sqrt{-g} \varphi_{A}^{, \mu}\right)-\frac{1}{2} \frac{\partial h_{B C}}{\partial \varphi^{A}} \varphi_{, \mu}^{C} \varphi_{, \nu}^{B} g^{\mu \nu}+W_{, A}=0,
$$

where $W_{, A}=\frac{\partial W}{\partial \varphi^{A}}$.

\section{The chiral cosmological model}

If we will consider a self-gravitating NSM with the potential of self-interaction in a homogeneous and isotropic universe, we arrive at the so-called [26] chiral cosmological model.

Now we consider the two-component nonlinear sigma model with the diagonal target space metric

$$
d s_{t s}^{2}=d \phi^{2}+h_{22}(\phi, \psi) d \psi^{2}
$$

In terms of the chosen target space (51), the energy-momentum tensor (2) can be presented in the following form

$$
T_{\mu \nu}=\phi_{\mu} \phi_{\nu}+h_{22} \psi_{\mu} \psi_{\nu}-g_{\mu \nu}\left[\frac{1}{2} \phi_{\rho} \phi^{\rho}+\frac{1}{2} h_{22} \psi_{\rho} \psi^{\rho}-W(\varphi, \psi)\right]
$$

Let us choose the metric of the homogeneous and isotropic universe in the Friedmann-Robertson-Walker (FRW) form

$$
d s^{2}=d t^{2}-a(t)^{2}\left(\frac{d r^{2}}{1-K r^{2}}+r^{2} d \theta^{2}+r^{2} \sin ^{2} \theta d \varphi^{2}\right)
$$

The chiral field equations for the two-component NSM (5) in the FRW universe (7) can be represented as

$$
\begin{aligned}
\ddot{\phi}+3 H \dot{\phi}-\frac{1}{2} \frac{\partial h_{22}}{\partial \phi} \dot{\psi}^{2}+\frac{\partial W}{\partial \phi} & =0 \\
3 H\left(h_{22} \dot{\psi}\right)+\partial_{t}\left(h_{22} \dot{\psi}\right)-\frac{1}{2} \frac{\partial h_{22}}{\partial \psi} \dot{\psi}^{2}+\frac{\partial W}{\partial \psi} & =0
\end{aligned}
$$

Einstein's equations can the be written in the form

$$
\begin{array}{r}
H^{2}=\frac{\kappa}{3}\left[\frac{1}{2} \dot{\phi}^{2}+\frac{1}{2} h_{22} \dot{\psi}^{2}+W\right]-\frac{K}{a^{2}} \\
\dot{H}=-\kappa\left[\frac{1}{2} \dot{\phi}^{2}+\frac{1}{2} h_{22} \dot{\psi}^{2}\right]+\frac{K}{a^{2}}
\end{array}
$$

The overdot means a derivative with respect to the time variable $t$. The Raychaudhuri field equation takes the form

$$
\frac{\ddot{a}}{a}=-\frac{\kappa}{3}\left[\dot{\phi}^{2}+h_{22} \dot{\psi}^{2}-W\right]
$$

From the Einstein and chiral field equations of the two-component NSM, we notice that all features of the EmU scenario will be present if the potential of $W(\phi, \psi)$ will contain the emergent potential $V(\phi)$ [2] for example $W(\phi, \psi)=$ $V_{E m U}(\phi)+\tilde{W}(\psi)$.

Let us consider now the two limiting cases, namely when $t \rightarrow-\infty$ and when $t \rightarrow \infty$. 


\section{Dynamics around the minimum}

Following the EmU scenario, let us consider the universe near the minimum $a_{\text {min }}=a_{i} \equiv a\left(t_{i}\right)$, where $t_{i} \rightarrow-\infty$. The only difference we suggest is that $\ddot{a} \neq 0, \ddot{a}>0$. This presentation of the dynamics in a small local region, when time is close to $t_{i}$, provides the possibility of studying the behaviour of the chiral fields $\phi, \psi$ and the target space metric coefficients $h_{22}$ in the EmU scenario.

The Einstein equations will take the following form (when $a=a_{i}=$ const, $\dot{a}=$ $0, \ddot{a}>0)$

$$
\begin{array}{r}
\frac{1}{2} \dot{\phi}^{2}+\frac{1}{2} h_{22} \dot{\psi}^{2}+W=\frac{3 K}{\kappa a^{2}} \\
\left.\frac{\ddot{a}}{a}\right|_{i}=-\kappa\left[\frac{1}{2} \dot{\phi}^{2}+\frac{1}{2} h_{22} \dot{\psi}^{2}\right]+\frac{K}{a^{2}}
\end{array}
$$

Here the index "i" is omitted.

The chiral field equations take the following form

$$
\begin{array}{r}
\ddot{\phi}-\frac{1}{2} \frac{\partial h_{22}}{\partial \phi} \dot{\psi}^{2}+\frac{\partial W}{\partial \phi}=0 \\
\partial_{t}\left(h_{22} \dot{\psi}\right)-\frac{1}{2} \frac{\partial h_{22}}{\partial \psi} \dot{\psi}^{2}+\frac{\partial W}{\partial \psi}=0
\end{array}
$$

For the scale factor, we take the form [4]

$$
a(t)=a_{i}\left(\beta+e^{\alpha t}\right) .
$$

Our first task will be to find an example of the solution with any potential which will support the stage under consideration. With this aim, let us simplify the equations (13][16). Let $h_{22}$ be functions of $\phi$ only. If $h_{22}$ will be an even function of $\phi$ then the equations (13]16) possess the symmetry $\phi \leftrightarrow-\phi, \psi \leftrightarrow$ $-\psi$. Therefore we will keep only the positive sign for the chiral fields. The resulting solution obtained under the above assumptions can be presented by the formulae:

$$
\begin{array}{r}
\phi=\sqrt{\frac{2 \lambda}{\kappa}} \int \sqrt{a_{i}^{-2}-\alpha^{2} e^{\alpha t}} d t=\sqrt{\frac{2 \lambda}{\kappa}}\left(2 F(t, \alpha)+A^{2} \ln \left[\frac{F-A^{2}}{F+A^{2}}\right]\right) \\
\lambda=\text { const, } A^{2}=\left(a_{i} \alpha\right)^{-2}, \quad F(t, \alpha)=\sqrt{A^{2}-\exp (\alpha t)} \\
\psi=C_{1} t, C_{1}=\text { const } \\
h_{22}=\frac{2(1-\lambda)}{\kappa C_{1}^{2}}\left(a_{i}^{-2}-\alpha^{2} e^{\alpha t}\right)=\frac{2 \alpha^{2}(1-\lambda)}{\kappa C_{1}^{2}} F^{2}(t, \alpha) \\
W=\frac{1}{\kappa}\left[\alpha^{2} e^{\alpha t}+\frac{2}{a_{i}^{2}}\right]=W_{1}(\phi)+W_{2}(\psi)+W_{*},
\end{array}
$$

where

$$
\begin{array}{r}
W_{2}(\psi)=\frac{2 \alpha^{2}}{\kappa}(1-\lambda) e^{\alpha \psi / C_{1}}, \\
W_{1}=\frac{\alpha^{2}}{\kappa}(2 \lambda-1) e^{\alpha t}, \\
W_{*}=\frac{2}{\kappa a_{i}^{2}}=\text { const }
\end{array}
$$


It is clear that it is impossible to present the direct dependence on $\phi$ for $h_{22}$ and $W_{1}$ in formulas (20, 23). Asymptotically, when $t \rightarrow-\infty$, inflaton $\phi \rightarrow-\infty$, target space coefficient $h_{22} \rightarrow \frac{2(1-\lambda)}{\kappa a_{i}^{2} C_{1}^{2}}$, the part of the potential $W_{1} \rightarrow 0$. It needs to be remembered that all obtained functions concern the period around $t_{i}$ and formally should be provided by index " $i$ ".

Thus we showed that the asymptotically Einstein static solution in the infinite past can be supported by the two chiral fields with geometric interaction by means of NSM. The differences with the original model of Ellis and Maartens are the following. If we consider the first chiral field $\phi$ as the inflaton and the $W_{1}$ part of the potential $W$ as the inflaton potential then we conclude that the potential started from zero. This is directly opposite to the original EmU scenario. But the presence of the second chiral field $\psi$ may give another way of comparison our EmU model with the original one. Namely, we have the possibility to choose the sign of integration constant $C_{1}$. If we set $C_{1}<0$ then the potential $W_{2}(\psi)$ tends to infinity with $\psi \rightarrow-\infty$ as in the original model with inflaton. In this case we can redefine the second field as the inflaton. In this situation another field will be responsible for geometric interactions because this field is included in the metric of the target space $h_{22}$.

The choice of integration constant $C_{1}>0$ will represent a new situation in the infinite past. As we have already mentioned for the obtained solution (18)-(24):

$$
\phi \rightarrow-\infty, \quad W_{1}(\phi) \rightarrow 0, \quad \psi \rightarrow-\infty, \quad W_{2}(\psi) \rightarrow 0, \quad h_{22} \rightarrow \frac{2(1-\lambda)}{\kappa a_{i}^{2} C_{1}^{2}}
$$

This means that the geometrical interaction with constant value of target space metric coefficient $h_{22}$ will support the Einstein static regime instead of the potential with enormous large value at the infinite past. Note that we are keeping $\ddot{a}_{i} \neq 0$ which was not considered in the original model. For the solution presented we have

$$
\ddot{a}_{i}=a_{i} \alpha^{2} \exp (\alpha t)>0
$$

\section{$5 \quad$ Evolution at late times}

To consider the EmU model at late times, we will solve the self-consistent system of the Einstein and chiral field equations (8,11) with the scale factor

$$
a(t)=\beta \exp \alpha t
$$

An investigation of this epoch is also connected with our attempts to understand the behaviour of the target space metric coefficient $h_{22}$ and the potential of selfinteraction $W(\phi, \psi)$.

\subsection{Solution A}

The solution under the assumption $h_{22}=h_{22}(\phi)$ is the following:

$$
\begin{array}{r}
\phi=-\frac{1}{\alpha \beta} \sqrt{\frac{2(1-\lambda)}{\kappa}} e^{-\alpha t}, \\
\psi=C_{2} t, \quad C_{2}=\text { const }
\end{array}
$$




$$
\begin{array}{r}
h_{22}=\frac{2 \lambda}{\kappa \beta^{2} C_{2}^{2}} e^{-2 \alpha t}=\frac{\lambda \alpha^{2}}{C_{2}^{2}(1-\lambda)} \phi^{2} \\
W=\frac{1}{\kappa}\left[3 \alpha^{2}+\frac{2}{\beta^{2}} e^{-2 \alpha t}\right]=W_{1}(\phi)+W_{2}(\psi)+W_{*},
\end{array}
$$

where

$$
\begin{array}{r}
W_{2}(\psi)=\frac{\lambda}{\beta^{2} \kappa} e^{-2 \alpha \psi / C_{2}}, \\
W_{1}=\frac{\alpha^{2}(2-\lambda)}{2(1-\lambda)} \phi^{2}, \\
W_{*}=\frac{3 \alpha^{2}}{\kappa}=\mathrm{const}
\end{array}
$$

In this case we obtain the direct formulae for the potential and for $h_{22}$. The dependence of $W$ on $\phi$ provides a possibility to consider a very physical situation, viz., a massive scalar field with the mass $m_{i n f l}^{2}=\frac{\alpha^{2}(2-\lambda)}{(1-\lambda)}$ which supports the epoch of late times in the EmU. It is clear from (27) and (30) that the influence of the second field $\psi$ will not be important when $t \rightarrow \infty$.

\subsection{Solution B}

We can obtain another solution under the same assumption for $h_{22}: h_{22}=$ $h_{22}(\phi)$. The solution is the following:

$$
\begin{array}{r}
\phi=-\frac{1}{\alpha \beta} \sqrt{\frac{2(1-\lambda)}{\kappa}} e^{-\alpha t}, \\
\psi=-\frac{1}{s} e^{-s t}, \quad s=\text { const, } \quad s \neq 0, \quad s \neq \alpha \\
h_{22}=\frac{2 \lambda}{\kappa \beta^{2}} e^{2(s-\alpha) t}=\frac{2 \lambda}{\kappa \beta^{2}} B_{*} \phi^{\frac{2(\alpha-s)}{\alpha}} \\
W=\frac{1}{\kappa}\left[3 \alpha^{2}+\frac{2}{\beta^{2}} e^{-2 \alpha t}\right]=W_{1}(\phi)+W_{2}(\psi)+W_{*},
\end{array}
$$

where

$$
\begin{array}{r}
W_{2}(\psi)=\frac{\lambda s(s+\alpha)}{\alpha \beta^{2} \kappa}(-s)^{2 \alpha / s-1} \psi^{2 \alpha / s}, \\
W_{1}=\frac{\alpha}{2(1-\lambda)}\{2 \alpha-\lambda(\alpha+s)\} \phi^{2}, \\
W_{*}=\frac{3 \alpha^{2}}{\kappa}=\text { const }
\end{array}
$$

The constant $B_{*}$ in 35 can be written as

$$
B_{*}=\left[-\sqrt{\frac{2(1-\lambda)}{\kappa}} \frac{1}{\alpha \beta}\right]^{2(s-\alpha) / \alpha}
$$

The evolution of the scalar field $\phi$ with time is the same as for the solution $\mathbf{A}$, but the "effective" mass of this field is given by

$$
m_{\text {infl }}^{2}=\frac{\alpha}{(1-\lambda)}\{2 \alpha-\lambda(\alpha+s)\}
$$


The second field $\psi$ also acquires an "effective" mass, but with a dependence on $\psi$, since $s \neq \alpha$.

\section{Conclusions}

We have studied the Emergent Universe scenario within the context of a nonlinear sigma model, and have shown that all the essential features of the Emergent Universe are present in our case. Since we know very little about the equation of state of ordinary matter under extreme conditions, our description provides an alternative scenario.

For the first time we investigated the dynamics of the source of the EmU around the minimum of the scale factor when $t \rightarrow-\infty$. The potential in this asymptotic case is proportional to the exponent of both the fields $\phi$ and $\psi$.

Analysis of the evolution of the chiral field at late times gives a new result for the potential form. Our two exact solutions show that the evolution of the EmU at late times can be supported by a massive inflaton field, while the auxiliary chiral field has exponential or power law dependence. This form of the potential tells us about the presence of the massive inflaton with some additional field during the inflationary stage in the EmU. Thus the obtained solutions for two limiting cases provide us some physical content about the chiral fields and their interaction by means of the kinetic term $h_{22}$.

We have obtained two examples of exact solutions for late times with a de Sitter expansion phase. Therefore we can consider the time of exit from inflation $t=t_{\text {end }}$ as the limiting case for the solutions. This time gives us restrictions on the parameters of the solutions. As future work we wish to extend the exact inflation method 28] for obtaining the key cosmological parameters the for nonlinear sigma model. this will enable us tio compare features from the presented solutions $\mathrm{A}$ and $\mathrm{B}$ with observation data.

\section{Acknowledgments}

SC is grateful to the University of KwaZulu-Natal, the University of Zululand and the National Institute for Theoretical Physics of South Africa for financial assistance and hospitality during his visit to these universities. SC is partly supported by the Russian Foundation for Basic Research (Project N 08-0291307). SDM acknowledges that this work is based on research by the South African Research Chair Initiative of the Department of Science and Technology and the National Research Foundation.

\section{References}

[1] Ellis G F R and Maartens R 2004 Class. Quant Grav. 21223

[2] Ellis G F R, Murugan J and Tsagas C 2004 Class. Quant Grav. 21233

[3] Mukherjee S, Paul B S, Maharaj S D and Beesham A 2005 Emergent universe in Starobinsky model arXiv: gr-qc/0505103 
[4] Mukherjee S, Paul B S, Dadhich N K, Maharaj S D and Beesham A 2006 Class. Quantum Grav. 236927

[5] Del Campo S, Herrera R and Labrana P 2007 Emergent universe in a Jordan-Brans-Dicke theory arXiv: 0711.1559 [gr-qc]

[6] Banerjee A, Bandyopadhyay T and Chakraborty S 2007 Emergent universe in Brane World Scenario arXiv: 0705.39333 [gr-qc]

[7] Schwinger J 1957 Ann. Phys. 2407

[8] Skyrme T H R 1958 Proc. Roy. Soc. A 247, 260

[9] Gell-Mann M, Levy M 1960 Nuovo Cim. 26, 705

[10] Perelomov A M 1987 Phys. Rep. 146, 136

[11] De'Alfaro V, Fubini S, Furlan G 1979 Nuovo Cim. A 50, 523

[12] Ivanov G G 1983 Theor. Math. Phys. 5745

[13] Chervon S V 1983 Izv. Vuz. Fiz. (Russ. Phys. J., New York) 26, 89

[14] Chervon S V 1995 Grav. \& Cosmol. 1, 91

[15] Chervon S V 1997 Grav. \& Cosmol. 3, No 2, 145

[16] Chervon S V 1995 J. Astrophs. Astron. 16, (Supplement series) 65

[17] Matzner R A and Misner C W 1967 Phys. Rev. 1541229

Chervon S V and Muslimov A G 1989 Phys. Lett. A 14214

Chervon S V and Shabalkin D Yu Grav. \& Cosmol. 6, 41 (2000)

[18] Lidsey J E, Wands D and Copeland E J 2000 Phys. Rept. 337343 arXiv: hep-th/9909061

[19] Matos T and Urena-Lopez F S 2000 Class. Quantum Grav. 17 L71

F S Urena-Lopez and Matos T 2000 Phys. Rev. D 62081302

Chervon S V 2008 Dark energy in sigma model presentation. $13^{\text {th }}$ Russian Gravitational Conference - International Conference on Gravitation, Cosmology and Astrophysics (RUSGRAV-13), People Friendship University of Russia, Moscow, Russia, 23-28 June 2008, Abstracts, p.127

[20] Barrow J D 1994, Phys. Rev. D49 3055.

[21] Ellis G R F and Madsen M 1991 Class.Quantum Grav. 8667

[22] Chervon S V, Zhuravlev V M and Shchigolev V K 1997 Phys. Lett. B 398, 269

[23] Zhuravlev V M, Chervon S V and.Shchigolev V K 1998 JETF, New York 87223

[24] Zhuravlev V M and Chervon S V 2000 JETF, New York 91227 
[25] Chervon S V 2001Exact solutions in standard and chiral inflationary models. Proceedings of 9th Marcel Grossmann Conference, Roma, 2000. World Scientific, 2001, p.1909-2000.

[26] Chervon S V 2002 Grav. \& Cosmol. 20, 145

[27] Chervon S V 2002 Int. J. Mod. Phys. A 17, 4451

[28] Chervon S V and Fomin 2008 I V Grav. \& Cosmol. 14163 